\title{
La profondeur et le fond: des concepts simples chez Lambert
}

Depth and Bottom: Simple Concepts by Lambert

\section{Arnaud Pelletier}

\section{Q OpenEdition}

\section{Journals}

Édition électronique

URL : https://journals.openedition.org/cps/672

DOI : $10.4000 / c p s .672$

ISSN : 2648-6334

Éditeur

Presses universitaires de Strasbourg

\section{Édition imprimée}

Date de publication : 27 novembre 2018

Pagination : 55-76

ISBN : 979-1-03440-024-9

ISSN : $1254-5740$

Référence électronique

Arnaud Pelletier, «La profondeur et le fond : des concepts simples chez Lambert », Les Cahiers

philosophiques de Strasbourg [En ligne], 44 | 2018, mis en ligne le 15 décembre 2018, consulté le 08

novembre 2022. URL : http://journals.openedition.org/cps/672 ; DOI : https://doi.org/10.4000/cps.672

\section{(c) (i) (2)(2)}

Creative Commons - Attribution - Pas d'Utilisation Commerciale - Partage dans les Mêmes Conditions 4.0 International - CC BY-NC-SA 4.0

https://creativecommons.org/licenses/by-nc-sa/4.0/ 


\title{
La profondeur et le fond: des concepts simples chez Lambert
}

\author{
Arnaud Pelletier
}

\section{L'hommage de Kant à Lambert}

Il est bien connu que Kant avait d'abord pensé dédier l'ouvrage qui allait devenir la Critique de la raison pure à Johann Heinrich Lambert, avec lequel il entretint une brève correspondance philosophique entre 1765 et 1770 . Dans la dédicace, rédigée en marge de son exemplaire de la Métaphysique de Baumgarten, Kant indique que l'ouvrage à paraitre est redevable des nombreuses suggestions et demandes du dédicataire, qui n'est pas nommé, et de son effort pour parvenir à «un concept de la méthode de la philosophie pure ${ }^{1}$. On conjecture souvent, à la suite d'Herman Jan de Vleeschauwer puis de Lewis White Beck, que Kant aurait en effet reconnu dans la dernière lettre de Lambert une suggestion menant à la première formulation du problème critique $^{2}$. En effet,

1 Kant, Réflexion 5024 (1776-78); AA (= Kant's Gesammelte Schriften, Akademie-Ausgabe, Berlin: De Gruyter), 18, 64.

2 H. J. De Vleeschauwer, "L'année 1771 dans l'histoire de la pensée de Kant", Revue belge de philologie et d'histoire, 3/3-4, 1934, p. 713-732; L. W. Beck, Early German philosophy: Kant and his predecessors, Cambridge/ London, 1969, p. 402-412; Essays on Kant and Hume, New Haven/ London, p. 107 sq. Alison LaYwine présente une autre justification de cette dédicace: "Lambert gave Kant the interesting idea that geometrical postulates in Euclid's sense were somehow essential to the reform of metaphysics they both sought» ("Kant and Lambert on geometrical postulates in the reform of metaphysics", in M. Friedman, M. Domski \& M. Dickson (éd.), Discourse on a New Method: Reinvigorating the Marriage of History and Philosophy of Science, Chicago: Open Court, 2010, 113-134). 
dans la lettre du 13 octobre 1770, Lambert adresse à Kant un certain nombre de remarques sur la Dissertation inaugurale que ce dernier vient de défendre et de lui faire parvenir. Lambert note entre autres choses:

"Je ne voulais pas que l'ontologie soit calquée sur les différentes parties de la métaphysique. [...] Il est utile dans l'ontologie de traiter des concepts empruntés de l'apparence (die vom Schein geborgte Begriffe) puisque la théorie de l'ontologie doit en fin de compte s'appliquer à nouveau aux phénomènes $n^{3}$.

Lambert soulève ainsi la question de l'application des concepts de l'ontologie à des objets de l'expérience. Sans explicitement exclure toute validité objective non phénoménale aux concepts de l'ontologie, Lambert suggère explicitement que c'est la méthode de l'astronome peut-être celle de Copernic - qu'il faut suivre en métaphysique:

«Ainsi l'astronomie commence-t-elle par le phénomène, en déduit sa théorie cosmologique et l'applique de nouveau, dans ses éphémérides, aux phénomènes et à leur prévision. Dans la métaphysique où l'apparence fait tant de difficulté, la méthode des astronomes sera sans doute la plus sûre $»^{4}$.

Si l'on s'en tient à cette reconstruction d'un premier pas vers le problème critique, l'hommage à Lambert ne devrait, directement, pas grand chose à la doctrine du Neues Organon ou de l'Anlage zur Architectonic. De fait, Kant semble bien avoir manqué, ou du moins ignoré, la singularité d'un aspect central de la réflexion lambertienne: à savoir la détermination des concepts fondamentaux ou catégories.

Il est en effet tout aussi connu que Kant a affirmé avoir profondément renouvelé la signification des catégories qui n'auraient survécu jusqu’à lui que sous la forme désuette et scolaire d'un lexique aristotélicien incapable d'épeler les phénomènes ni les pensées. De fait, il leur attribue un rôle et une tâche inédites en tant que concepts purs a priori de l'entendement $(\mathrm{A} 79 / \mathrm{B} 104)^{5}$; il prétend donner une justification de leur énumération complète (A81/B107); et il se contente de renvoyer aux manuels scolaires en usage pour trouver une liste convenable des prédicables, c'est-à-dire des concepts qui peuvent être dérivés de ces concepts-

3 Lambert à Kant du 13 octobre 1770; AA 10, 108.

4 Ibid. Et L. W. BecK, Essays, op. cit., p. 110.

5 Nous renvoyons, selon l'usage, aux deux éditions originales (A et B) de la Critique de la raison pure. 
souches, puisque tel n'est pas là l'essentiel du problème catégorial ${ }^{6}$. D'un mot, la doctrine des concepts-souches (Stammbegriffe, A81/B107) - ou concepts fondamentaux (Grundbegriffe) - de l'entendement ne saurait être confondue avec la doctrine des prédicaments conçus comme chefs généraux de toute synthèse prédicative. Cela ne fait aucun doute; mais cela ne permet en aucune manière d'affirmer que personne ne se serait préoccupé avant Kant du sens selon lequel des concepts peuvent être dits fondamentaux ni de la manière dont il est possible de les identifier.

C'est en effet très précisément le reproche que Johann Heinrich Lambert avait déjà formulé à l'encontre de Baumgarten dans la préface de l'Anlage zur Architectonic:

«Au cours de mes recherches, le manuel de Baumgarten s'est révélé m'être de moins en moins utile. Baumgarten donne des définitions de ses concepts ontologiques mais pas grand chose de plus. Qui plus est, il ne définit la plupart du temps que des mots isolés et rarement des concepts exprimés par une locution complète. Aussi me restait-il encore une question à éclaircir, à savoir: d'où viennent les concepts [ontologiques], comment les obtient-on et à quoi servent-ils vraiment en fin de compte?».

Le reproche de Lambert à l'encontre de Baumgarten est massif: Baumgarten ne cesse de multiplier des définitions dans sa Metaphysica mais oublie de déterminer en quel sens certains concepts sont fondamentaux du fait qu'il pense parvenir à des concepts généraux et

6 Voir les Prolégomènes (AA 04, 325) où Kant renvoie à la Métaphysique de Baumgarten pour une liste convenable des prédicables.

7 J. H. LAmbert, Anlage zur Architectonic, oder Theorie des Ersten und des Einfachen in der philosophischen und mathematischen Erkenntniß, Riga, 1771 (désormais: Anlage), Vorrede, V: «Das Baumgartensche Compendium wurde mir waehrenden Untersuchungen immer unbrauchbarer. Baumgarten giebt Definitionen von seinen ontologischen Begriffen und nicht viel anders. Ueberdies sind mehrentheils nur einzelne Wörter, selten die in ganzen Redensarten liegende Begriffe definirt worden. Auch blieb mir immer die Frage: woher die Begriffe sind, wie man dazu gelange, und wohin sie endlich unmittelbar dienen? selbst zu erörtern». Le manuel d'Alexander Gottlieb Baumgarten dont il s'agit est la Metaphysica (Halle, 1739), remanié deux fois en 1743 et 1750, et réédité de nombreuses fois ensuite $(1757,1763$, 1768, 1779). Voir aussi: W. STARK, «Kant und Baumgarten: Exemplare der Metaphysica. Ein nachfragender Bericht", Editio. Internationales Jahrbuch für Editionswissenschaft, 27/1, 2014, p. 96-111. 
purs - censés être les plus fondamentaux - par une simple analyse. Ainsi au $\$ 685$ de l'Anlage:

«Baumgarten comprend la pureté de l'entendement (puritas intellectus) comme un plus grand degré de profondeur [Tiefsinnigkeit], et ne la pose donc que comme relative puisqu'en réalité la pureté est une unité qui n'admet en toute rigueur que des fractions - ainsi l'eau, l'argent ou l'or sont bien purs mais peuvent être mélangés à des choses étrangères selon une infinité de degrés. C'est l'absence d'images étrangères et d'apparences sensibles qui constitue [de manière caractéristique] l'entendement pur, mais Baumgarten cherchait à y parvenir par l'analyse des concepts ${ }^{8}$.

Autrement dit, la profondeur (Tiefsinngkeit) de l'analyse des concepts n'atteint jamais le fond de l'entendement pur ni les concepts véritablement fondamentaux - précisément parce qu'elle les considère de manière isolée pour en donner des définitions, au lieu de les comprendre au sein de «locutions complètes", à savoir au sein des axiomes et des postulats. La pureté ne s'obtient pas par analyse. Ni par abstraction d'ailleurs: c'est ainsi que Christian Wolff lui-même a pu reconnaître qu'il n'y a jamais, pour nous, d'usage entièrement pur de l'entendement et de la raison du seul fait que ces facultés sont limitées et ne peuvent atteindre le fond des choses:

«Maintenant, parce que notre entendement n'est jamais entièrement pur, il ne peut non plus atteindre le plus haut degré de perspicacité. Et c'est pourquoi nous ne pouvons parvenir au fond (ergründen) de tout ce qu'il y a dans les choses: car nous disons que nous parvenons au fond de quelque chose quand nous comprenons distinctement tout ce qu'il y a en elle»?.

8 Anlage, $\$ 685$ : «Baumgarten sieht die Reinheit des Verstandes (Puritas intellectus), als einen groeßern Grad von Tiefsinnigkeit an, und setzet sie demnach nur relativ, da doch die Reinheit (Puritas) eine Einheit ist, die schlechthin nur Brueche admittirt, wie z. E. Wasser, Silber, Gold etc. nicht mehr als rein, hingegen durch unzaehlige Stufen mit fremdem Zeuge vermenget seyn kann. Das Wegfeyn fremder Bilder und des sinnlichen Scheines machet den reinen Verstand aus. Baumgarten aber suchte denfelben durch das Analysiren der Begriffe zu erhalten".

9 C. Wolff, Vernünfftige Gedancken von Gott, der Welt und der Seele des Menschen, auch allen Dingen urberhaupt [Métaphysique allemande], Frankfurt/Leipzig, 1733, \$852: «Weil nun unser Verstand niemahls ganz rein ist, so kan er auch den höchsten Grad der Scharfsinnigkeit 
Toujours est-il que le reproche, adressé par Lambert à l'encontre de Baumgarten, d'un défaut de saisie du fondamental est particulièrement frontal puisque ce dernier caractérisait précisément l'ontologie de science fondamentale, Grund-Wissenschaft, en tant qu'elle est la science des prédicats généraux de l'étant et des premiers principes de la connaissance $^{10}$, tout comme le wolffien Gottsched la caractérisera de Grundlehre ${ }^{11}$. Bref, la science prétendument fondamentale n'atteint pas au fondamental.

Au delà de la formulation générale d'un projet commun de «réforme de la méthode de la métaphysique» tel qu’il apparaît dans leur correspondance $^{12}$, Lambert a ainsi précisément formulé à l'encontre de Baumgarten un reproche que Kant formulera à l'encontre de Wolff: leur manie de tout définir - leur pruritus definiendi - n'aboutit qu'à une "orgueilleuse ontologie» qui recouvre, élude ou ignore la question $\mathrm{du}$ fondamental. Kant louera certes, selon une formule bien connue, «l'esprit de profondeur» de Wolff mais c'est pour lui reprocher par ailleurs, dans ses leçons de logique, d'avoir «initié cette manie de définir (Definirsucht) en philosophie ${ }^{13}$. En somme, Lambert et Kant ont posé le même diagnostic: la manière 'wolffo-baumgartenienne' d'établir des concepts ontologiques les aurait détournés du sens même des concepts fondamentaux. Et elle a surtout eu pour effet de transformer la métaphysique en un simple lexique de mots, incapable d'épeler les phénomènes, et qui ne pouvait que devenir l'objet de moqueries et de mépris ${ }^{14}$. Ce diagnostic commun aurait pu à lui seul justifier que Kant

nicht erreichen. Und daher können wir nicht alles, was in denen Dingen ist, ergründen: denn wir sagen, daß wir etwas ergründen, wenn wir alles deutlich begreiffen, was in ihm anzutreffen ist".

10 Cf. A. G. Baumgarten, Metaphysica, Halle: Hemmerde, 1779, $\$ 1$ : «Metaphysica est scientia primorum in humana cognitione principiorum»; et \$4: «Ontologia (Grund-Wissenschaft) est scientia praedicatorum entis generalium».

11 Cf. Johann Christoph GotTsched, Erste Gründe der gesamten Weltweisheit, Leipzig: Breitkopf, 1733.

12 Cf. M. Friedmann, Kant's construction of nature. A Reading of the Metaphysical Foundations of Natural Science, Cambridge, 2013, p. 121-122.

13 Kant, Logik Philippi; AA 24.1, 460.

14 Voir l'Anlage, $\$ 4$, à propos des successeurs d'Aristote: «Sie verwandelten seine Metaphysic in ein Regifter von Woertern, Unterscheidungen und Fragen, welche faemmtlich mehr dieneten, die menschliche Erkenntniß 
rende hommage à Lambert. Mais laissons-là les reconstructions de leurs relations philosophiques pour envisager maintenant le problème des concepts fondamentaux chez Lambert.

\section{Le défaut du fondamental}

Dès la préface du Neues Organon, publié en 1764, Lambert tient pour équivalents les concepts fondamentaux et les concepts simples de notre connaissance (einfachen oder Grundbegriffen unserer Erkenntnis) ${ }^{15}$. Cet usage lexical s'oppose à ceux qui ont confondu les concepts fondamentaux avec les concepts généraux - fussent-ils generalissima déterminés comme les plus hauts genres d'une ligne prédicamentale. Le reproche s'adresse en particulier à la méthode dichotomique par genres et espèces empruntée par la Metaphysica de Baumgarten. Si la construction de la Metaphysica suit le manuel wolffien de Ludwig Philipp Thümmig ${ }^{16}$, l'originalité propre de Baumgarten est de proposer une sorte de tabulation exhaustive en multipliant les oppositions qui permettent de quadriller non les aspects du réel mais les prédicats de l'étant ${ }^{17}$ : Baumgarten établit ainsi des propositions disjonctives dont il élimine ensuite l'un ou l'autre membre (ainsi «la substance est soit simple soit composée» n'est pas une thèse soutenue par Baumgarten mais une proposition disjonctive

dunkeler, verworrener und ungewisser zu machen, als ihre allgemeine Gruende in ein helleres Licht zu setzen, und sie der Anwendung naeher zu bringen. So bliebe die Metaphyic viele Jahrhunderte, und wurde endlich zum Gegenftande des Gespoettes und der Verachtung».

15 J. H. Lambert, Neues Organon oder Gedanken über die Erforschung und Bezeichung des Wahren und dessen Unterscheidung vom Irrthum und Schein, Leipzig: Wendler, 1764 (désormais: Neues organon), Vorrede, p. 12.

$16 C f$. Ludwig Philipp Тнӥмmig, Institutiones philosophiae wolfianae, Frankfurt: Renger, 1725.

17 Cf. A. G. Baumgarten, Metaphysica, 1779, \$6: "Ontologia continet praedicata entis I) interna 1) universalia, quae sunt in singulis, 2) disiunctiva, quorum alterutrum est in singulis; II) relativa». Le caractère systématique de l'œuvre de Baumgarten est signalé dès la monographie d'Eberstein, Versuch einer Geschichte der Logik und Metaphysik bey den Deutschen von Leibniz bis auf gegenwärtige Zeit, Halle, 1794, p. 225. 
résultant de la combinaison de concepts antérieurs dont il va écarter le second membre) ${ }^{18}$.

Lambert distingue cependant, au $\$ 523$ de l'Anlage, deux manières pour parvenir à la distinction et la complétude des concepts: «la voie de l'abstraction [par genres et espèces] et celle de l'analyse (Auflösen)». L'abstraction ne parvient à des propositions générales (allgemein) que par ressemblance (Ähnlichkeit); l'analyse permet de parvenir à des possibilités universelles et inconditionnées - c'est-à-dire aussi non limitées (uneingeschränkt):

«Le premier genre de généralité (Allgemeinheit) se rapporte au sujet, de sorte que l'on dise: tous les $A$ sont $B$. Mais l'autre se rapporte au prédicat, de sorte que l'on dise: $A$ peut, en toute modification de $B$, être $B$. La ressemblance des choses est en elle-même purement idéale, et n'est ainsi pas la raison (Grund) de la possibilité des choses, mais cette dernière a ses propres raisons (Gründe) et elle s'enracine, lorsqu'elle ne doit pas être limitée, dans ce qui est simple. Que l'on procède ainsi en science et l'on aura un point de départ (Anfang) à partir duquel, comme ce doit être dans le royaume de la vérité, les concepts composés se présenteront comme des prédicats plutôt que comme des sujets. Mais ce n'est pas ainsi que l'on a procédé en métaphysique [jusqu'ici] où l'on a d'emblée considéré que le concept d'une chose était un sujet qui était si composé que son analyse ne peut guère être achevée. Et selon toute apparence, on ne peut achever cette analyse si l'on doit rechercher tous les fundamenta divisionis. La division des choses en genres et espèces n'est qu'un ordre purement local; par contre, l'ordre légal commence par les possibilités simples et inconditionnées, et est par là complètement différent. Aussi, il est tout à fait possible dans ce dernier cas d'avancer pas à pas (Schritt für Schritt), alors que dans le premier cas, on ne rencontre un ordre que partiellement (Stückweise) mais un désordre absolu dans l'ensemble $»^{19}$.

18 Sur ce point, nous renvoyons à notre étude: «Monades sans monadologie? La Métaphysique de Baumgarten face à l'exposé monadologique de Leibniz", Studia Leibnitiana, 45/2, 2013, p. 209-227.

19 Anlage, $\$ 523$ : «Die erstere Art von Allgemeinheit geht auf das Subject, so daß man saget: Alle $A$ find $B$. Die andere aber auf das Praedicat, so daß man saget: $A$ kann, nach jeden Modificationen des $B, B$ seyn. Die Aehnlichkeit der Dinge ist an sich schlechthin ideal ( $\$ 372.164 .425$.$) , und in so fern$ ist sie nicht der Grund von der Moeglichkeit der Dinge, sondern diese hat ihre eigene Gruende, und faengt, wo sie uneingeschraenkt seyn soll, bey 
Le premier sens du fondamental s'esquisse ainsi comme celui de la possibilité inconditionnée de concepts simples en tant que fondements de la possibilité des concepts composés. Ces fondements déterminent un ordre légal (gesetzlich), c'est-à-dire ici un ordre général des choses mêmes, qui se distingue de l'ordre purement local des divisions arbitraires et idéales. Dans le Neues organon, Lambert distingue alors deux sens de la simplicité: la simplicité relative des concepts plus simples que d'autres; et la simplicité absolue des concepts qui ne peuvent pas être davantage analysés - une question qui, dit-il, «a une certaine ressemblance avec la question de la divisibilité de la matière $»^{20}$. Autrement dit, la profondeur des degrés de généralité des concepts ne suffit pas assurer le fondement de leur possibilité.

Dans sa Versuch einer Geschichte der Logik und Metaphysik bey den Deutschen von Leibniz bis auf gegenwärtige Zeit, publiée en 1794, Wilhelm Ludwig Gottlob von Eberstein reproche à Lambert d'avoir confondu les concepts simples (einfache Begriffe) de notre pensée avec des concepts originairement simples (ursprünglich einfache Begriffe) ${ }^{21}$.

dem einfachen an, (Alethiologie $\$$ 239. 246. 250.). Thut man dieses in dem wissenschaftlichen Vortrage, so wird man einen Anfang haben, und die zufammengesetzten Begriffe werden darinn, wie in dem Reiche der Wahrheit (Alethiol. \$241.), als Praedicate vorkommen, ehe sie als Subjecte vorkommen. So aber verfuhr man in der Metaphysic nicht, sondern man nahm den Begriff eines Dinges gleich anfangs als Subject vor, welcher, wie wir vorhin gesehen haben ( $\$ 521.522$.$) , so sehr zusammengesetzet ist, daß$ man des Analysirens kaum ein Ende findet. Und allem Ansehen nach findet man gar keines, wenn man alle Fundamenta diuifionis aufsuchen soll, $(\$ 520.184$. 247.). Die Eintheilungen der Dinge in Arten und Gattungen ist gleichsam eine bloß locale Ordnung ( $\$ 338$.), dahingegen die gesetzliche bey den einfachen und unbedingten Moeglichkeiten anfaengt, und eben dadurch einen ganz andern Weg geht. Es ist daher gar wohl moeglich, daß, da man bey der letztern anfangen und Schritt fuer Schritt fortgehen kann, bey der erstern hingegen die Ordnung nur stueckweise, im Ganzen aber schlechthin eine absolute Unordnung vorkomme, (\$181.)».

20 Cf. Neues Organon, II (Aléthiologie), $\$$ 6: «Ob aber ein Begriff, so einfach er auch seyn mag, sich nicht immer in noch einfachere aufloesen lasse, ist eine ganz andre Frage, die mit der Frage von der Theilbarkeit der Materie eine gewisse Aehnlichkeit hat, aber auch mehr oder minder davon verschieden ist".

21 Cf. Eberstein, op. cit. (note 16), p. 299: "Auch scheint er nicht daran gedacht zu haben, daß die Ideen, welche für die Menschen einfach sind, noch nicht zu den ursprünglich einfachen gezählt werden können ». 
Eberstein manque le point: il s'agit de déterminer les fondements de notre pensabilité (Gedenkbarbeit) des choses en tant qu'ils en garantissent la possibilité. Ceci dit, Lambert s'expose bien à la difficulté de la voie des concepts logiquement inanalysables ou non dérivables. Ce problème des prédicats incomplexes a parfois été confondu avec le problème catégorial même si, pour Aristote, les catégories signifient les «dits-sansliaison" ou incomplexes mais ne leur sont pas identiques ${ }^{22}$. Toutefois, ils posent le problème des concepts fondamentaux du point de vue de leur appréhension conceptuelle. Comment Lambert résout-il cette difficulté?

\section{Les conceps simples}

La présentation la plus synthétique du projet lambertien se trouve dans la seconde partie de sa logique ou Neues Organon, intitulée "Aléthiologie ou doctrine de la vérité", et particulièrement dans les deux premiers chapitres. La première section est consacrée à la détermination des concepts simples ou pensables par soi (einfache oder für sich gedenkbare Begriffe). Les énoncés principaux du développement sont les suivants :

1. Toute vérité suppose que les concepts qui la constituent ne soient pas contradictoires $(\$ 2)$.

2. Seuls des concepts simples écartent absolument toute possibilité de contradiction - en tant qu'ils sont à eux-mêmes leurs propres composantes caractéristiques (Merkmale). Conformément à une remarque d'Aristote ${ }^{23}$, la simplicité de ces concepts les prémunit de toute contradiction interne, et par conséquent du faux $(\$ 4)$.

3. La possibilité des concepts simples a été établie dans la Dianoiologie selon un principe de compositionnalité dont la formule rappelle la thèse monadologique: «Il doit y avoir des concepts simples puisqu'il y en a de composés, et que c'est pour cette raison même qu'on les appelles composés» $(\$ 5)^{24}$.

22 Cf. Aristote, Catégories, IV, $1 \mathrm{~b} 25$.

23 Cf. Aristote, De Anima, III, 6, 430 a 26-27.

24 Neues organon, II, $\$ 5$ : «Es muesse einfache Begriffe geben, weil es zusammengesetzte giebt, weil diese eben deswegen zusammengesetzt heißen". 
4. Étant à eux-mêmes leurs propres marques, les concepts simples n'ont donc pas de diversité discursive interne et sont homogènes ou uniformes (einförmig), contrairement aux concepts abstraits. Il n'y a, par exemple, qu'un seul espace puisque toutes les parties de l'espace sont des parties d'un même espace toujours présupposée ${ }^{25}$; ou encore quelque chose existe ou n'existe pas mais il n'y a pas de degré d'intensité de l'existence $(\$ 12)$. C'est la raison pour laquelle les concepts simples sont aussi appelés des «unités absolues" ( $\$ 12)$.

5. Etant à eux-mêmes leurs propres marques, la saisie des concepts simples intervient dans la sensation (Empfinfung) $(\$ 14,16)$, ou plus exactement dans la conscience à l'occasion de la sensation (\$21) : ce sont des données immédiates de la conscience (unmittelbar aus dem Bewußtsein) $(\$ 9,21,24)$ qui ne peuvent être mises en doute par le sceptique.

Cet aspect signale immédiatement le statut ambivalent des concepts simples: leur simplicité est garantie par l'immédiateté de leur saisie dans l'expérience; mais leur simplicité garantit à son tour leur statut a priori indépendamment de toute expérience. Car il s'agit bien de chercher avant tout "d'où (woher) nous tenons les premiers fondements (Grundlage) de nos concepts, et dans quelle mesure il est en eux quelque chose de simple qui peut être alors considéré de manière a priori $»^{26}$. Autrement dit: la saisie des concepts simples intervient empiriquement; mais ils sont considérés comme a priori non en vertu de leur origine mais de la simplicité de leur contenu. Etant à eux-mêmes leurs propres marques, ces concepts ne sont susceptibles d'aucune définition réelle, mais leur contenu représentationnel ne peut être explicité qu'à travers une sensation (immédiate) - de sorte qu'ils peuvent jouer le rôle de fondements pour des définitions réelles et des connaissances a priori. En somme, «les concepts simples, que nous obtenons au moyen des sens et de la conscience, constituent les fondements de notre connaissance» (\$53).

Le caractère d'immédiateté à la conscience permet à Lambert d'identifier un certain nombre de ces concepts simples. C'est le cas du

25 Kant reprendra cet argument pour établir l'unicité et le caractère non discursif de l'espace dans l'Esthétique transcendantale (A24-25/B38-40).

26 Neues organon, II, $\$ 21$ : «Hier aber ist der Ort nicht, dieses vorzunehmen, weil wir hier eigentlich nur unterfuchen, woher wir die erste Grundlage zu unsern Begriffen haben, und wiefern etwas einfaches darinn ist, welches sich sodann als a priori ansehen lasse». 
concept même de conscience puisque nous sommes immédiatement conscients que nous n'aurions aucune sensation ni concept sans la conscience $(\$ 70)$. L'existence est aussi un concept simple: nous avons une conscience immédiate de notre propre existence ( $\$ 24)$. C'est le cas aussi de l'unité, dont nous avons conscience immédiate dans le mot «je» et dans la représentation de tout concept $(\$ 26)$. Le concept de force motrice est connu immédiatement par le toucher (Gefühl) - de même que les concepts de pression et de résistance - et ce indépendammant du degré de pression ou de résistance dont on peut faire l'expérience $(\$ 18)$. Le concept de temps compte aussi parmi les concepts simples puisqu'il vient immédiatement de la succession des sensations, pensées, etc. $(\$ 23)$.

En fin de compte, Lambert donne une liste (incomplète) de dix concepts qui se trouvent, poursuit-il, avoir déjà été identifiées par Locke comme des concepts simples (einfache Begriffe): l'étendue, la solidité, le mouvement, l'existence, la durée et la succession, l'unité, la conscience, la force motrice, la volontê2 ${ }^{2}$. En effet, le critère phénoménologique d'une immédiateté de la saisie de concepts simples (donc uniformes) chez Lambert semble bien reprendre le critère lockéen de l'apparition et, plus précisément, de la donnée phénoménologique de certaines idées d'une manière simple (ou non composée), laquelle rend raison de l'uniformité de leur contenu représentationnel:

"Les idées que les qualités produisent par les sens dans l'esprit y entrent simples et sans mélange. [...] Et rien n'est plus manifeste pour un homme que sa perception claire et distincte de ces idées simples, dont chacune, exempte en elle-même de composition, ne renferme rien d'autre qu'une seule manifestation uniforme, qu'une seule conception dans l'esprit, et ne saurait être distinguée en idées différentes ${ }^{28}$.

27 Neues organon, II, $\$ 36$ : «1. Die Ausdehnung. 2. Die Soliditaet. 3. Die Bewegung. 4. Die Existenz. 5. Die Dauer und Succession. 6. Die Einheit. 7. Das Bewußtseyn. 8. Die Kraft zu bewegen. 9. Das Wollen». En II, 68, Lambert dissocie la durée et la succession et élimine la redondance entre mouvement et force motrice: "1. Bewußtsein, 2. Existenz, 3. Einheit, 4. Dauer, 5. Sukzession, 6. Wollen, 7. Solidität, 8. Ausdehnung, 9. Bewegung, 10. Kraft».

28 Locke, Essai sur l'entendement humain, II, ii, 1, trad. J.-M. Vienne, Paris: Vrin, 2001, p. 187-188. 
La simplicité relève exclusivement du mode d'apparaître - au demeurant passif - de l'idée à la conscience. Elle ne relève ni des qualités objectives qui les suscitent (et c'est ainsi qu'il y a des idées simples tant des qualités premières des corps - comme l'étendue, la solidité, le mouvement que des qualités secondes de la perception des corps, comme celles des couleur ${ }^{29}$ ) ni des différentes origines des idées. Ainsi, parmi les idées simples mentionnées par Lambert, Locke considère que l'idée de solidité est suscitée par un seul sens (le toucher); l'étendue et le mouvement, quoique simples, tirent leur origine de plusieurs sens conjoints; les idées de conscience (perception) et de volonté sont quant à elles obtenues par réflexion (qu'il ne faut pas confondre avec le processus d'abstraction); et les idées d'existence et d'unité viennent à la fois de la sensation et de la réflexion ${ }^{30}$. Cette simplicité dans l'apparence de l'idée garantit la distinction de l'idée selon Locke - un point que Leibniz a bien entendu contesté. Mais indépendamment du débat au sujet des qualités logiques (distinctes ou confuses) qu'il faut attribuer aux idées simples, et particulièrement aux idées des sens, il n'en reste pas moins que, selon Locke, et en raison même de la dépendance de la réflexion par rapport à la sensation, toutes les idées simples ont leur origine a posteriori dans l'expérience. Or nous avons déjà noté que Lambert attribue aux concepts simples un statut a priori indépendamment de toute expérience. Aussi, bien que la caractérisation du simple puisse être commune avec celle de Locke, et bien que Lambert renvoie à une liste lockéenne des idées simples, il s'écarte cependant de «l'anatomie des concepts et de la connaissance» que ce dernier met en œuvre, a posteriori, à partir des choses données empiriquement sans envisager une synthèse a priori des concepts simples - c'est-à-dire sans envisager des pensées universelles qui ne résulteraient pas de l'abstraction ${ }^{31}$. La

29 Ibid., II, viii, 9.

30 Ibid., II, vii.

31 Cf. Neues organon, II, 29: "Locke begnuegte sich naemlich, sein ganzes Werk auf Erfahrungssaetze zu bauen, und geht demnach durchaus a posteriori, weil er schlechthin die Sachen nimmt, wie sie sind. Wir nennen daher sein System eine Anatomie unsrer Begriffe und Erkenntniß, weil er ungefehr eben fo verfaehrt, wie die Anatomici sich einen Begriff der innern und einfachern Theile des Leibes und ihrer Verbindung zu machen suchen. Dieses ist nun hier unsre Absicht nicht. Wir haben in der Dianoiologie gesehen, was die wissenschaftliche Erkenntniß, und so weit sie a priori 
distinction opérée par Lambert entre l'anatomie de Locke et le procédé a priori de la science ne peut manquer de rappeler la manière dont Kant distinguera lui-même le processus de la déduction transcendantale de la simple dérivation physiologique de Locke ${ }^{32}$. Toujours est-il que Lambert prend soin d'indiquer dès la préface qu'il n'avait pas encore lu Locke que la moitié du Neues Organon était déjà achevée ${ }^{33}$ - ce qui est une manière de ménager la spécificité de son projet par rapport à l'auteur anglais, et de réserver à la synthèse des concepts simples un second sens du fondamental.

\section{Les axiomes (Grundsätze) des concepts simples}

Le deuxième chapitre de l'aléthiologie porte sur les principes, c'està-dire les postulats et les axiomes (Forderungen et Grundsätze) que les concepts simples donnent ${ }^{34}$. Les concepts simples n'importent ainsi pas tant en eux-mêmes que par les principes qu'ils permettent d'établir et qui

gehen kann, vor der gemeinen und bloß historischen Erkenntniß voraus habe. Diesen Vortheilen wuerden wir nicht naeher kommen, wenn wir uns schlechthin bey der Anatomie unsrer Begriffe aufhalten wuerden. Es ist nicht genug, einfache Begriffe ausgelesen zu haben, sondern wir muessen auch sehen, woher wir in Ansehung ihrer Zusammensetzung allgemeine Moeglichkeiten (Dianoiol. $\$ 692 s q$.) aufbringen koennen». Voir aussi l'Anlage, I, $\mathbb{\$} 7$ : «Und in dieser Absicht kann man sagen, daß Locke die menschlichen Begriffe anatomirt, Leibnitz aber dieselben analysirt habe».

32 KANT, CRP, $\$ 13$ (A 87/B119): "Une telle recherche des premiers efforts de notre faculté de connaître pour s'élever des perceptions singulières à des concepts généraux possède sans nul doute une grande utilité, et il faut savoir gré au célèbre Locke d'avoir été le premier à ouvrir la voie. Reste qu'une déduction des concepts purs a priori ne peut jamais être menée à bien sur ce mode, dans la mesure où, vis à vis de leur usage futur, qui doit être totalement indépendant de l'expérience, il faut que ces concepts produisent un tout autre acte de naissance que celui qui atteste leur dérivation à partir des expériences. Une telle tentative de dérivation physiologique ne peut pas du tout s'appeler déduction ".

33 Neues organon, préface: «Und zwar muß ich hier fagen, daß ich Lockens Werke erst nachgesehen, nachdem ich bereits die erste Helfte dieses Hauptstueckes geschrieben ".

34 Le titre du chapitre est «Des Axiomes et postulats que les concepts simples nous donnent" (Von den Grundsaetzen und Forderungen, so die einfachen Begriffe angeben), que Lambert reformule au $\$$ 67: «welche Grundsaetze und Postulata diese einfache Begriffe uns angeben»). 
viennent formuler un certain nombre de lois de la pensabilité qui ne sont rien d'autre que des fondements des sciences a priori. Lambert pense ainsi pouvoir dépasser les lacunes de la méthode de Wolff, lequel suivait la méthode d'Euclide dans l'établissement des définitions, mais laissait totalement de côté l'usage des axiomes et des postulats chez Euclide. Or, pour Lambert, postulats et axiomes sont plus fondamentaux encore que les concepts simples dans la mesure où ils garantissent la possibilité des choses mêmes. Aussi le projet lambertien est-il distingué tant du projet lockien que du projet wolffien en ce qu'il associe des aspects de l'un et de l'autre, à savoir les concepts simples et l'abstraction d'un côté et la possibilité d'une science a priori de l'autre: «Dans le deuxième chapitre de l'Aléthiologie, j'associe les concepts simples de Locke à la méthode de Wolff, et je fournis ainsi le fondement de diverses sciences qui sont, au sens strict, a priori $\aleph^{35}$.

Quel est le statut de ces Grundsätze qui, d'un côté, présupposent des concepts simples et, de l'autre, interviennent au fondement des sciences?

Concernant leur rapport aux concepts simples, Lambert assure que les Grundsätze n'en sont pas dérivés mais partagent avec eux leur caractère de simplicité du fait qu'ils ne sont que des modifications et des possibilités des concepts simples. Ainsi écrit-il au $\$ 124$ de l'Aléthiologie:

"Nous avons de la même manière présenté les axiomes et les postulats tout simplement comme tels, et nous avons en cela suivi Euclide. Ces axiomes et ces postulats ne sont pas dérivés de définitions et, autant que je le comprenne, ne doivent pas l'être non plus. Car les définitions servent soit à rendre un concept distinct, soit à déterminer son extension. Mais cela est sans objet dans le cas des concepts simples puisqu'ils sont à eux-mêmes leurs propres caractères internes unifiés et qu'ils ne peuvent donc être plus clairs précisément parce qu’ils sont simples. Or puisque les axiomes n'exposent que certaines modifications des concepts simples, et les postulats certaines de leurs possibilités, il est manifeste que ces modifications et possibilités sont également simples en elles-mêmes

35 Neues organon, préface: "In dem zweyten Hauptstuecke der Alethiologie verbinde ich Lockens einfache Begriffe mit Wolfens Methode, und bringe dadurch die Grundlage zu verschiedenen Wissenschaften heraus, die im strengsten Verstande a priori sind». 
et sont données clairement et immédiatement avec les concepts simples ${ }^{36}$.

Puisque Lambert avait caractérisé la simplicité des concepts simples par l'immédiateté de leur saisie intellectuelle, on peut comprendre que les principes - axiomes et postulats - puissent aussi être simples s'ils sont donnés en même temps qu'eux. La simplicité de la saisie n'implique en effet pas l'unicité atomique du contenu saisi, mais le fait que ce contenu soit intérieurement unifié (einig) de sorte que la saisie en soit simple et non composée. Mais l'on peut alors se demander s'il n'existe pas un rapport d'antériorité et de composition entre les concepts simples et les principes qu'ils donnent: en quel sens comprendre que les axiomes sont des modifications des concepts simples? Et ces axiomes sont-ils fondamentaux de la même manière?

Tournons-nous donc vers l'exposé des axiomes des concepts de solidité, d'existence et de force dans l'Anlage zur Architectonic, que Lambert a publié avec l'aide de $\mathrm{Kant}^{37}$, et qui est plus développé que celui du Neues Organon. En effet, là où le Neues organon distingue trois axiomes de la solidité, l'Anlage énonce cinq axiomes donnés par le concept d'un solide matériel:

1. Un solide remplit un espace autant qu'il peut.

2. Un solide exclut d'autres solides du lieu où il est.

3. Un solide a les trois dimensions de l'espace.

4. L'espace ne peut être rien de plus que rempli par des solides.

36 Neues organon, $\$ 164$ : «\$124. Ferner haben wir die Grundsaetze und Postulata ebenfalls schlechthin als solche angegeben, und hierinn sind wir dem Euclid gefolgt. Diese Grundsaetze und Postulata sind nicht erst aus Definitionen hergeleitet, und so viel ich begreife, sollen sie es auch nicht seyn. Denn die Definitionen dienen entweder den Begriff deutlich zu machen, oder seinen Umfang zu bestimmen. Beydes faellt bey einfachen Begriffen weg, weil diese sich selbst ihr eigenes und einiges inneres Merkmaal sind, und folglich nicht mehr als klar seyn koennen, eben deswegen, weil sie einfach sind. Da nun die Grundsaetze gewisse Modificationen, die Postulata aber gewisse Moelichkeiten bey den einfachen Begriffen anzeigen, so ist offenbar, daß diese Modificationen und Moeglichkeiten an sich auch einfach sind, und zugleich auch mit dem einfachen Begriffe klar und zugegeben werden ".

37 Kant à Lambert, du 31 décembre 1765; AA 10, 54-57. 
5. Un solide a une densité absolue, et est par conséquent une unité inaltérable ${ }^{38}$.

Les Grundsätze de la force motrice (bewegende Kraft) sont les suivants:

1. Un solide est de lui-même au repos, ou sans mouvement.

2. Un solide est mis en mouvement par un autre solide.

3. Tout changement dans le mouvement d'un solide a pour cause un autre solide qui est en contact avec le solide en mouvement.

4. Dans un espace libre, un solide mis une fois en mouvement conserve sa direction et sa vitesse.

5. Le mouvement est fonction de la force qui met le solide en mouvement et suit la direction selon laquelle la force est appliquée ${ }^{39}$. Enfin, les sept Grundsätze de l'existence sont formulés comme suit:

1. L'existence est une unité absolument inaltérable.

2. Sans des solides et des forces, ou sans rien de substantiel en général, rien n'existe.

3. Ce qui existe a une durée.

4. Ce qui existe est en un lieu.

5. Un même solide n'existe pas en même temps dans plus d'un lieu.

6. Différents solides n'existent pas en même temps dans le même lieu.

7. Ce qui existe n'est pas divers en même temps, ou ce qui existe est une seule et même chose (numero idem) ${ }^{40}$.

38 Anlage, $\$ 88$ : «Der Begriff der Soliditaet giebt uns ebenfalls einige Grundsaetze, die bey dem materiellen Soliden ohne Widerrede angewandt werden. $1^{\circ}$. Das Solide fuellet einen Raum aus, so weit es geht. $2^{\circ}$. Das Solide schleußt anderes Solides von dem Orte aus, da es ist. $3^{\circ}$. Das Solide hat die drey Dimensionen des Raumes. $4^{\circ}$. Der Raum kann mit Solidem nicht mehr als ausgefuellet seyn. $5^{\circ}$. Das Solide hat eine absolute Dichtigkeit, und daher ist es eine Einheit, die unveraenderlich ist, $(\$ 91)$ ».

39 Anlage, $\$ 94: \ll 1^{\circ}$. Das Solide ist an sich in Ruhe, oder ohne Bewegung. $2^{\circ}$. Das Solide wird durch anderes Solides in Bewegung gesetzt. $3^{\circ}$. Jede Aenderung in der Bewegung des Soliden wird durch anderes Solides verursacht, welches das bewegte Solide unmittelbar beruehret. $4^{\circ}$. Im freyen Raume behaelt das einmal in Bewegung gesetzte Solide seine Richtung und Geschwindigkeit. $5^{\circ}$. Die Bewegung ist in Verhaeltniß der Kraft, womit das Solide in Bewegung gesetzet wird, und folget der Richtung, nach welcher die Kraft angebracht wird». Les axiomes 4 et 5 reprennent les deux premières lois de Newton.

40 Anlage, $\$ 103:$ : $1^{\circ}$. Die Existenz ist eine absolute unveraenderliche Einheit. $2^{\circ}$. Ohne Solides und Kraefte, oder ueberhaupt ohne etwas Substantiales 
L'ordre de la présentation est significatif puisque les axiomes de la solidité (\$88) interviennent dans ceux de la force motrice $(\$ 94)$ et de l'existence $(\$ 103)$. D'un autre côté, il est patent que les axiomes de l'existence - et en particulier de l'existence en un lieu de l'espace ( $\$ 103.4 .5 .6)$ - rend raison du fait qu'un solide, en tant qu'il remplit un espace et occupe un lieu, doit exclure d'autres solides du lieu où il est (\$ 88.2). Autrement dit, l'impénétrabilité du corps ou plutôt du solide (88.2) est une propriété liée à qui existe réellement dans un espace - une proposition qui sera contestée par Kant dans les Principes métaphysiques de la science de la nature ${ }^{41}$. Cette même impénétrabilité est supposée dans les trois axiomes de la force motrice $(\$ 94)$. Toutefois le premier axiome de la solidité (88.1 : «Un solide remplit un espace autant qu'il peut») est, lui aussi, impliqué par le deuxième et, de fait, par tous les autres. Tous les axiomes de la solidité ne sont donc pas premiers par rapport aux autres, mais le premier l'est: on peut de nouveau faire le parallèle avec Locke, pour qui le concept de solidité est impliqué dans un certain nombre de concepts issus de la sensation, sans pour autant que ces derniers en dérivent - puisqu' "il n'y a pas d'idée que nous recevions de la sensation avec plus de constance que la solidité « ${ }^{42}$. Pourtant, malgré leur fonction fondamentale particulière parmi les Grundsätze, Lambert note que les axiomes de la solidité pourraient bien être révisés:

"Nous avons le concept de solidité par le toucher, mais ce dernier ne nous en donne pas les différences internes. Dans le concept que nous en avons, il semble qu'il n'y ait pas d'impossiblité à ce que le solide

existirt nichts, $(\$ 60.90.) 3^{\circ}$. Was existirt dauert. $4^{\circ}$. Das Existirende ist an einem Orte. $5^{\circ}$. Einerley Solides existirt nicht zugleich an mehr als einem Orte. $6^{\circ}$. Verschiedenes Solides existirt nicht zugleich an einem Orte. $7^{\circ}$. Was existirt, ist nicht zugleich verschieden, oder was existirt ist ein und eben dasselbe, (numero idem)».

41 Kant, Principes métaphysiques de la science de la nature; AA 04, 497-498: "Lambert et d'autres ont appelé solidité la propriété de la matière de remplir un espace et veulent qu'il faille la supposer en tout ce qui existe, du moins dans le monde sensible extérieur. Selon leur concept, la présence réelle de quelque chose dans l'espace doit déjà en elle-même par son concept, et partant conformément au principe de contradiction, impliquer cette résistance».

42 Locke, Essai sur l'entendement humain, II, iv, 1. 
ne puisse avoir différents degrés de densité interne ${ }^{43}$. Les axiomes qui ont été introduits devraient ainsi être modifiés de telle sorte que la solidité ne soit plus une unité absolue et inaltérable, ou qu'un espace rempli le puisse être par des solides plus ou moins denses, etc. $»^{44}$.

Le concept fondamental d'une absolue impénétabilité des solides est ainsi susceptible d'être révisé, Lambert ne présentant ici que les axiomes liés à ce concept en tant qu'il a un usage dans les sciences de la nature et, partant, en tant qu'il pourra également être abandonné. On retrouve ici l'ambivalence qui était constitutive des concepts simples et qui était rendue explicite dans le projet d'allier Locke et Wolff: les axiomes ont une origine a posteriori mais doivent valoir a priori. Une telle concession peut sembler rhédibitoire: alors que Lambert annonçait que les fondements métaphysiques devaient être inaltérables comme la véritée $^{45}$, il laisse désormais ouverte la possibilité d'un changement de ces fondements. On peut comprendre que Kant n'ait pas suivi Lambert dans cette manière de réformer la méthode de la métaphysique. Toutefois, cette concession n'est peut-être pas seulement le signe d'une lacune qu'il faudrait éviter, mais l'indice que les Grundsätze ne touchent pas encore le fond des concepts simples. Et, en effet, Lambert identifie des concepts encore plus fondamentaux pour penser le passage de l'un à l'autre: les concepts relationnels.

\section{Les concepts de relation et l'amphibologie du fondamental}

Après l'énoncé des axiomes et des postulats, Lambert note aux paragraphes 110 et 111 de l'Aléthiologie qu'il existe des concepts encore plus simples et plus généraux:

43 Ce qui contreviendrait à l'axiome 5 de la solidité: «Un solide a une densité absolue, et est par conséquent une unité inaltérable».

44 Anlage, \$91: «Wir haben den Begriff der Soliditaet durch das Gefuehl, und dieses giebt uns die innere Unterschiede desselben nicht an. In dem Begriffe den wir davon haben, scheint auch keine Unmoeglichkeit zu liegen, daß das Solide nicht verschiedene Grade der innern Dichtigkeit haben koenne. Dadurch wuerden die angefuehrten Grundsaetze in so weit geaendert, daß die Soliditaet keine absolute und unveraenderliche Einheit waere, daß ein ganz ausgefuellter Raum mit mehr oder minder dichtem Soliden ausgefuellet feyn koenne etc».

45 Anlage, $\$ 21$ : «Die Grundlehre soll unveraenderlich seyn, wie die Wahrheit ». 
"Nous nous tournons maintenant vers quelques concepts de relation, qui sont plus simples et plus généraux, du fait qu'ils nous servent précisément à passer d'un concept à un autre. Le premier de ces concepts est l'identité ou mêmeté et est opposé à la différence et au changement ${ }^{46}$.

Les concepts relationnels de l'identité et de la différence sont des concepts simples qui interviennent dans de nombreux axiomes: ils sont les premiers fondements de la connaissance (Grundlage unserer Erkenntnis, \$118) en tant qu'ils soutiennent le système complet "des concepts, axiomes et relations" qui définit le «système ou royaume de la vérité» ( $\$ 160)$, mais en tant également qu'ils donnent en fin de compte le principe d'identité et de contradiction (\$162-164). Il peut paraître étonnant que Lambert commence par reprocher à la métaphysique dogmatique de reposer sur le seul principe de contradiction et de négliger ainsi l'apport des sciences empiriques pour finalement conclure au rôle constitutif des concepts relationnels de l'identité et de la différence. Les concepts d'identité et de différence conservent leur caractère fondamental du fait qu'ils ne sont ni produits par comparaison à partir des choses, à la manière de Locke ${ }^{47}$, ni compris comme de simples prédicats externes ou relatifs des étants parmi d'autres, à la manière de Baumgarten ${ }^{48}$.

Sans entrer dans le détail foisonnant de l'Aléthiologie, il reste que la réponse apportée par Lambert aux difficultés de l'ontologie de Wolff et de Baumgarten fut de déterminer progressivement le fondamental au travers des Grundbegriffe, Grundsätze et Grundverhältnisse. Il accomplit ainsi deux gestes pour répondre aux défauts du fondamental. D'une part, si l'ontologie doit être comprise comme une doctrine fondamentale (Grundlehre) exposant le fondement (Grundlage) de notre connaissance, alors elle ne doit pas se contenter de définir un ensemble de concepts

46 Neues organon, II, $\$ 110-111$ : «Wir werden nun noch einige Verhaeltnißbegriffe vornehmen, die einfacher und allgemeiner sind, weil diese eigentlich dienen, uns von einem Begriff auf den andern zu fuehren. Der erste dieser Begriffe ist die Jdentitaet oder Einerleyheit, und diese wird der Verschiedenheit und Veraenderung entgegengesetzt».

47 Locke, Essai sur l'entendement humain, II, xxvii, 1.

48 Dans sa Metaphysica, Baumgarten ne signale la distinction de l'idem et du diversum qu'au titre des prédicats externes ou relatifs des étants, à côté du simultané et du successif, de la cause et du causé, du signe et du désigné (AA 17, 083-103). 
fondamentaux, mais elle doit exposer les axiomes (Grundsätze) et les postulats de la possibilité des choses que ces concepts subsument: tel serait le véritable achèvement de la méthode d'Euclide en métaphysique. D'autre part, au-delà des axiomes mêmes, il faut reconnaître qu'il y a certains concepts de relation qui sont plus fondamentaux encore que les concepts simples. Faut-il alors penser que Lambert réintroduit par un côté le dogmatisme métaphysique qu'il avait chassé de l'autre? Au contraire, en distinguant les Grundbegriffe des Grundverhältnisse (et en particulier ceux qui donnent lieu au principe de contradiction), Lambert signifie bien que les concepts fondamentaux sont de deux sortes, et que l'erreur de la métaphysique dogmatique a été de les confondre. De nouveau, un parallèle peut être effectué avec la doctrine critique de Kant, dans laquelle sont identifiés deux types de concepts fondamentaux dont la confusion - ou l'usage amphibologique - serait le vice originaire de la métaphysique dogmatique, à savoir: d'un côté, les catégories et, de l'autre, les concepts de la réflexion (parmi lesquels figurent les concepts d'identité et de diversité). Reprenons, pour finir, cette dernière distinction, qui se pourrait bien être un des héritages insoupçonnés de Lambert en Kant.

Dans le chapitre intitulé "Amphibologie des concepts de la réflexion" qui clôt l'Analytique transcendantale, Kant indique qu'il faut reconnaittre aux concepts de la réflexion un caractère fondamental du point de vue de la constitution subjective des concepts et des jugements - à savoir du fait qu'ils tiennent leur fonction de l'usage de l'entendement lui-même ${ }^{49}$; mais qu'il faut leur refuser du point de vue du contenu représentationnel (Inhaltsbezogenheit) dans la mesure où il ne sont que des modes d'appartenance des représentations les unes aux autres (Zueinandergehörigkeit). En effet, l'usage de la réflexion suppose qu'il existe deux types de relation (Verhältnis) ${ }^{50}$. D'un côté, il y a la relation des représentations entre elles qui n'est rien d'autre que leur comparaison (Vergleichung) selon les méta-concepts, ou concepts de

49 Cf. R. Malter, "Reflexionsbegriffe. Gedanken zu einer schwierigen Begriffsgattung und zu einem unausgeführten Lehrstück der Kritik der reinen Vernunft", Philosophia naturalis, 1982, p. 130-131.

50 Cf. CRP, A 260/B 316: "[La réflexion] est la conscience de la relation (Verhältniß) qui existe entre des représentations données et nos diverses sources de connaissance, laquelle peut seule déterminer exactement la relation (Verhältnißs) qu'elles ont entre elles». 
la réflexion, que Kant énumère: l'identité et la diversité, la convenance et la disconvenance, l'intérieur et l'extérieur, le déterminable et la détermination. La comparaison est donc une relation d'appartenance des représentations les unes aux autres: Kant la désigne par l'expression «zu einander gehören». Mais le sens de cette appartenance réciproque ne peut être exactement déterminé si l'on ne sait à quelle faculté ces représentations appartiennent d'abord toutes ensemble: c'est ainsi le rôle de la réflexion que de retrouver l'appartenance commune de ces représentations à l'une des deux sources de la connaissance, la sensibilité ou l'entendement. Cette relation d'appartenance des concepts à une source commune est désignée par l'expression «zusammen gehören zu». Les concepts de la réflexion décrivent ainsi les rapports fondamentaux entre les représentations, et sont fondamentaux au sens où ils précèdent les concepts des choses mêmes (die Vergleichung der Vorstellungen, welche vor dem Begriffe von Dingen vorhergeht, A 269/B 325). L'amphibologie, que dénonce Kant, consiste donc à confondre deux sortes de concepts fondamentaux, les concepts de la réflexion - concepts subjectifs qui s'appliquent tant aux représentations sensibles qu'aux représentations purement intellectuelles - et les catégories qui ont une validité objective. Ailleurs, Kant dira même que l'utilité du système des catégories consiste aussi «à repousser tous les concepts d'un genre différent [à savoir les concepts de la réflexion], qui autrement pourraient s'immiscer parmi les concepts purs de l'entendement ${ }^{51}$. S'il revient à Kant d'avoir identifié le motif d'une amphibologie des concepts de la réflexion; le motif d'une confusion des différents sens du fondamental fut cependant l'une des lignes directrices des traités de Johann Heinrich Lambert.

\section{Bibliographie}

Baumgarten Alexander Gottlieb, Metaphysica, Halle: Hemmerde, 1779.

Beck Lewis White, Early German philosophy: Kant and his predecessors, London: Oxford University Press, 1969.

Beck Lewis White, Essays on Kant and Hume, New Haven: Yale University Press, 1978.

51 Kant, Prolégomènes à toute métaphysique future qui pourra se présenter comme science, $\$ 39$; AA 04, 326. 
Eberstein W. L. G. Freyherr (von), Versuch einer Geschichte der Logik und Metaphysik bey den Deutschen von Leibniz bis auf gegenwärtige Zeit, Halle: J. G. Kuff, 1794.

Friedman Michael, Kant's construction of nature. A Reading of the Metaphysical Foundations of Natural Science, New-York: Cambridge University Press, 2013.

GotTsched Johann Christoph, Erste Gründe der gesamten Weltweisheit, Leipzig: Breitkopf, 1733.

Kant Immanuel, Gesammelte Schriften, Berlin (et alii): Reimer (et alii), 1902- (cité "AA» suivi du numéro de volume et de page).

LAmbert Johann Heinrich, Neues Organon oder Gedanken über die Erforschung und Bezeichung des Wahren und dessen Unterscheidung vom Irrthum und Schein, Leipzig: Wendler, 1764.

Lambert Johann Heinrich, Anlage zur Architectonic, oder Theorie des Ersten und des Einfachen in der philosophischen und mathematischen Erkenntniß, Riga: Hartknoch, 1771.

LaYwine, Alison, "Kant and Lambert on geometrical postulates in the reform of metaphysics", in M. Friedman, M. Domski \& M. Dickson (dir.), Discourse on a New Method: Reinvigorating the Marriage of History and Philosophy of Science, Chicago: Open Court, 2010, p. 113-134.

Locke John, Essai sur l'entendement humain, traduction Jean-Michel Vienne, Paris: Vrin, 2001.

Matter Rudolf, «Reflexionsbegriffe. Gedanken zu einer schwierigen Begriffsgattung und zu einem unausgeführten Lehrstück der Kritik der reinen Vernunft », Philosophia naturalis, 19, 1-2, 1982, p. $125-150$.

Pelletier Arnaud, «Monades sans monadologie? La Métaphysique de Baumgarten face à l'exposé monadologique de Leibniz», Studia Leibnitiana, 45/2, 2013, p. 209-227.

STARK, Werner «Kant und Baumgarten: Exemplare der Metaphysica. Ein nachfragender Bericht", Editio. Internationales Jahrbuch für Editionswissenschaft, 27/1, 2014, p. 96-111.

Tнüмmig Ludwig Philipp, Institutiones philosophiae wolfianae, Frankfurt: Renger, 1725.

Vleeschauwer Herman Jan (de), "L'année 1771 dans l'histoire de la pensée de Kant", Revue belge de philologie et d'histoire, 3, 3-4, 1934, p. 713-732.

WolfF Christian, Vernünfftige Gedancken von Gott, der Welt und der Seele des Menschen, auch allen Dingen urberhaupt [Métaphysique allemande], Frankfurt/Leipzig: Renger, 1733. 